Classification

Physics Abstracts

61.16Di $-61.50 \mathrm{Jr}-82.65 \mathrm{Dp}$

\title{
Investigations of the morphology of copper surfaces by scanning tunneling microscopy
}

\author{
J.C. Girard, S. Gauthier, S. Rousset and J. Klein
}

Groupe de Physique des Solides, Universités Paris 7 et 6, Laboratoire associé au CNRS, 2 Place Jussieu, 75251 Paris, France

(Received September 22, 1993; accepted November 02, 1993)

\begin{abstract}
Résumé. - Les principaux résultats de deux études de la morphologie de surfaces de cuivre sont présentés afin d'illustrer la capacité de cette technique à mettre en évidence les relations entre la morphologie de la surface et les mécanismes atomiques responsables de celle-ci. La première étude concerne les fluctuations de position de marches à l'équilibre sur des surfaces vicinales de la face (100). La seconde étude s'intéresse à la génération d'îlots de lacunes et d'adatomes par bombardement ionique sur la face (100) du cuivre.
\end{abstract}

\begin{abstract}
The main results of Scanning Tunneling Microscopy (STM) studies of the morphology of copper surfaces are presented. It illustrates the ability of this technique to investigate the relationship between surface morphology and the atomic mechanisms which determine it. The first study concerns the equilibrium fluctuations of steps on surfaces vicinal from (100), the second the vacancy and adatom islands generated by low energy ion bombardment on the (100) face of copper.
\end{abstract}

\section{Introduction.}

Crystallographic structure determination has been one of the main objectives of surface science in the last 30 years. The combined use of sophisticated experimental techniques, mainly low energy electron diffraction and powerful theoretical modelling methods has led to a good understanding of the crystallographic structure of the surfaces of many materials. The interest is now shifting toward the comprehension of the structure at scales larger than the size of the surface unit cell, that is, of surface morphology. One of the reasons for this evolution, besides the need to better control many processes of technological relevance, is the recent progress of experimental techniques, especially microscopies, which provide direct space imaging capabilities. Surfaces can now be visualised by low energy electron microscopy, transmission or reflection electron microscopy and novel local probe techniques, such as STM or various force microscopies. Among these techniques, STM, due to its ability to image at the atomic level on $(\mu \mathrm{m})^{2}$ areas, is well suited to investigate the relationship between surface morphology and the atomic mechanisms which determine it. 
To illustrate these considerations, we present in this paper the main results of STM investigations on surfaces of copper, the details of which will be published elsewhere $[1,2]$.

The first study concerns the behaviour of monoatomic steps in equilibrium on two vicinal faces of $\mathrm{Cu}(100)$, which differ mainly by the atomic structure of their steps. We will show that one can extract from the observations some of the energetic parameters governing the steps structure.

The second study is an investigation of the morphology of a surface during an out-of-equilibrium process, ionic sputtering. We will show that during ionic sputtering, vacancies are generated, which can diffuse and nucleate vacancy islands, making this process very similar to a growth experiment, vacancies and vacancy islands assuming the roles of adatoms and adatom islands respectively.

\section{Experimental.}

The experiments were conducted in an ultra-high vacuum chamber whose base pressure was in the low $10^{-10}$ Torr range. Tungsten tips were etched by electrochemical erosion and cleaned in situ prior to the experiment by resistive heating to approximately $1000^{\circ} \mathrm{C}$. The samples were cleaned by repeated cycles of argon ion abrasion ( $400-600 \mathrm{~V}, 10$ minutes), followed by annealing to $600^{\circ} \mathrm{C}$. All the images presented here were recorded at room temperature in the constant current mode. The tunneling current and the tunneling bias were respectively in the range 0.4-1 $\mathrm{nA}$ and a few hundred $\mathrm{mV}$. In general, the tunneling conditions are not critical on metals and as far as we are concerned do not affect the observed morphology.

\section{Step wandering on $\mathrm{Cu}(11,1,1)$ and $\mathrm{Cu}(8,1,0)$.}

In many cases, surface morphology can be described in terms of atomic steps, in the framework of the well-known T.L.K. (Terrace, Ledge, Kink) model [3]. This description is correct when the temperature is not too high and the steps not too close. In these conditions, one can neglect the elementary excitations of the terraces and the concept of a step remains well defined. One of the problems which has attracted wide interest is to understand the nature of the interactions between steps, because they control the orientational dependence of the surface free energy and thus the equilibrium shape of crystals near their facets [4].

At finite temperature, steps meander about their average position by excitation of kinks. On a stepped surface, these fluctuations are limited by step interactions, which originate from entropic or energetic contributions [5]. Simple models [6,7] show that the step behaviour is controlled by two energetic parameters. The amplitude of the fluctuations is determined by the stiffness of an isolated step, which in turn depends on the kink energy, and the intensity of the interaction potential of the steps. Both quantities can be extracted from the correlation function of the fluctuations along the step edge which reads [7] (Fig. 1):

$$
\left[<x(y)-x(0)>^{2}\right]=<x(0)^{2}>[1-\exp (-y / \xi)]
$$

with:

$$
<x(0)^{2}>=\left\{k T b^{2}(T) / 8 c(L) a_{\|}\right\}
$$

and:

$$
\xi=\left\{k T a_{\|} / 2 b^{2}(T) c(L)\right\}^{1 / 2}
$$




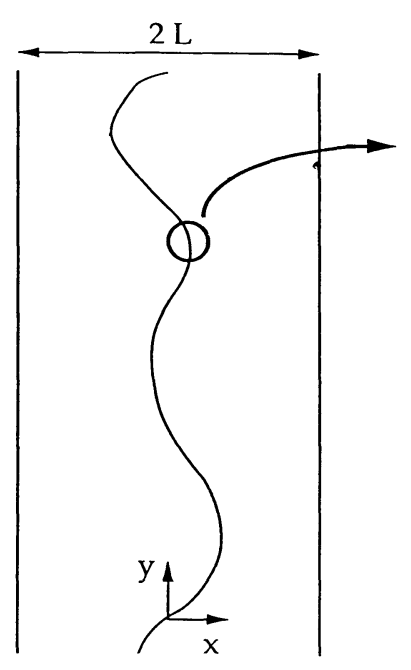

(a)

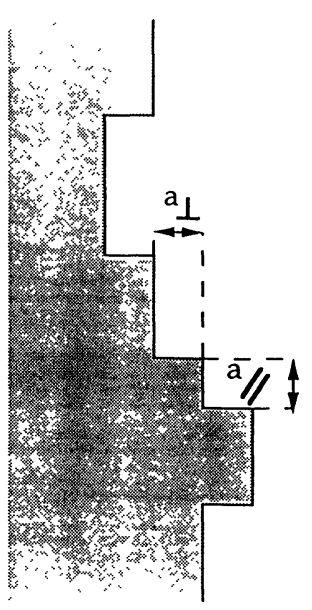

(b)

Fig. 1. - On the left, a step meandering between two straight steps separated by $2 L$. On the right, the kink structure of the step.

$c(L)$ being the concavity of the interaction potential. The spatial dependence of this potential has been questioned [8] but recent experiments $[9,10]$ tend to confirm theoretical predictions of a $1 / L^{2}$ decay. Assuming a potential $U(x)=A / x^{2}$ gives $c(L)=6 A / L^{4}$.

For $y<<\xi$ one gets:

$$
\left[<x(y)-x(0)>^{2}\right]=b^{2}(T) y / a_{\|}
$$

It can be shown that this expression describes the behaviour of an isolated step, by exploiting the analogy between a configuration of the step and a trajectory of a one dimensional random walk [11]. The diffusivity $b^{2}(T)$ is the mean square displacement in the direction perpendicular to the step for an interatomic distance $a_{\|}$along the step. The correlation length $\xi$ then characterises the crossover between the small scale fluctuations for which the step behaves as it were free and the large scale fluctuations which are dominated by the interaction with neighbouring steps.

It is thus in principle possible to extract these atomic quantities from images of stepped surfaces. This has been done recently on surfaces vicinal from $\mathrm{Si}(111)$ by reflection electron microscopy [9] and scanning tunneling microscopy [12].

We have performed similar measurements on copper $[13,14]$. One advantage of this material is that, in contrast to silicon, the mobility at room temperature is high enough to insure that the surface is in thermal equilibrium at room temperature, avoiding delicate measurements at high temperature to access equilibrium quantities. The $\mathrm{Cu}(11,1,1)$ and $\mathrm{Cu}(810)$ faces investigated are oriented at about $7^{\circ}$ from the (100) atomic plane in the [0-11] and [001] zone axis. They differ mainly by the structure of the steps edges which are dense [0-11] rows for $(11,1,1)$ and more open [001] rows for (810). In both cases, the terrace width is near $1.4 \mathrm{~nm}$.

The correlation function obtained from the image of the (810) face in figure $2 \mathrm{a}$ is displayed in figure $2 \mathrm{~b}$. As expected, it deviates for large $y$ from the linear behaviour observed for small $y$. The slope near the origin gives a diffusivity $b^{2}(T)=0.44 \AA^{2}$. The correlation length is $90 \AA$. The kink 
energy $E_{\text {kink }}(810)$ can be extracted from $b^{2}(T)$ by the relation:

$$
E_{\text {kink }}(810) / k_{\mathrm{B}}=T \ln \left\{a^{2} / 2 b^{2}(T)\right\}=590 \mathrm{~K}
$$
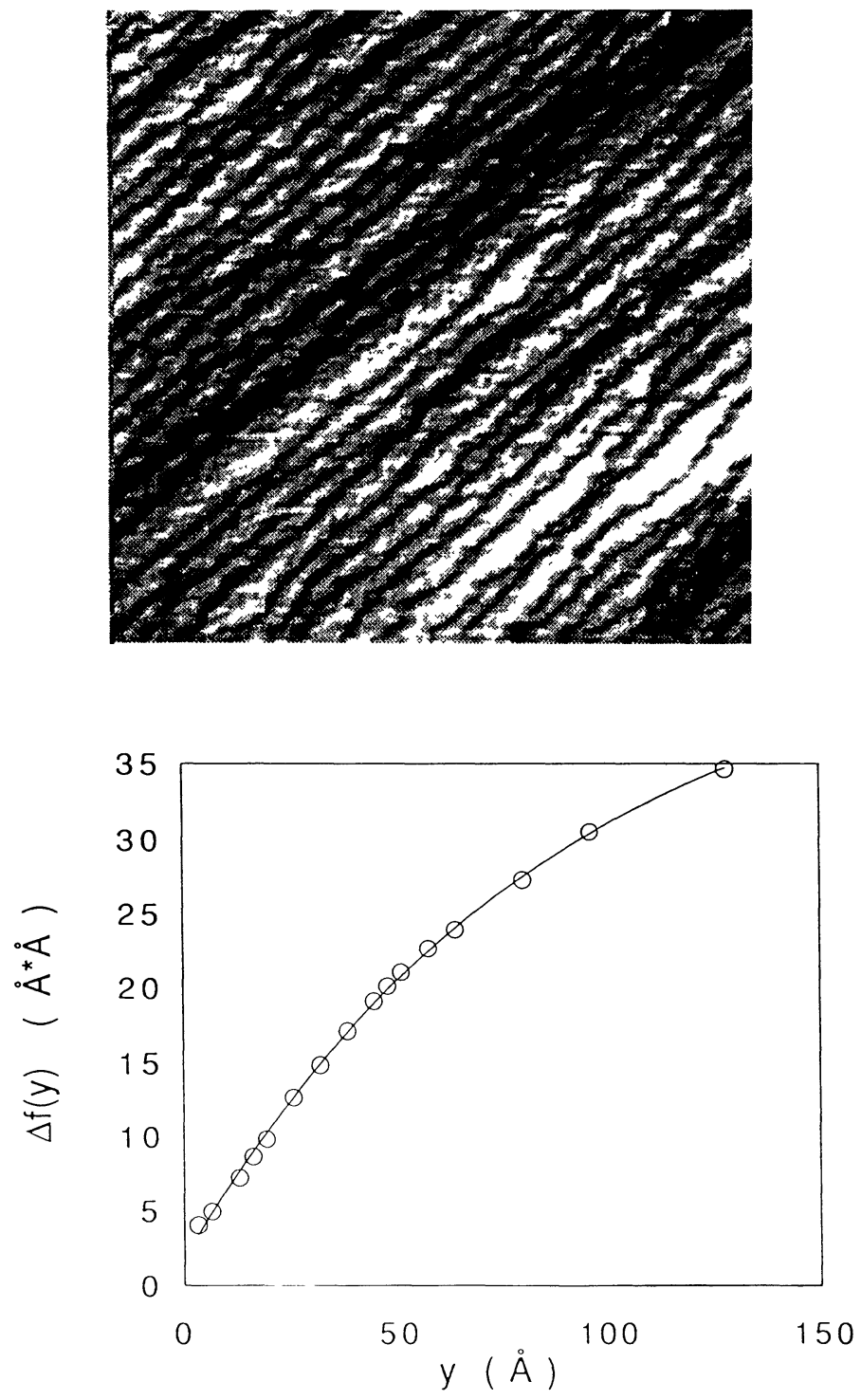

Fig. 2. $-516 \times 479 \AA$ image of the $\mathrm{Cu}(810)$ face with the corresponding correlation function. The quantity plotted is in fact the correlation function for the terrace width, which is $2 f(y)$ for non-correlated steps. The data were acquired from left to right, scanning horizontally with a scanning frequency of $0.8 \mathrm{~Hz}$.

The correlation function obtained from the image of the $(11,1,1)$ face in figure $3 \mathrm{a}$ is displayed in figure $3 \mathrm{~b}$. One does not observe, as on the (810) face, a significant deviation from a straight 
line. This indicates that the correlation length for this face is larger than the size of the image. One can only evaluate $b^{2}(T)=0.12 \AA^{2}$ leading to a kink energy:

$$
E_{\text {kink }}(11,1,1) / k_{\mathrm{B}}=T \ln \left\{2 a^{2} / b^{2}(T)\right\}=1380 \mathrm{~K} .
$$
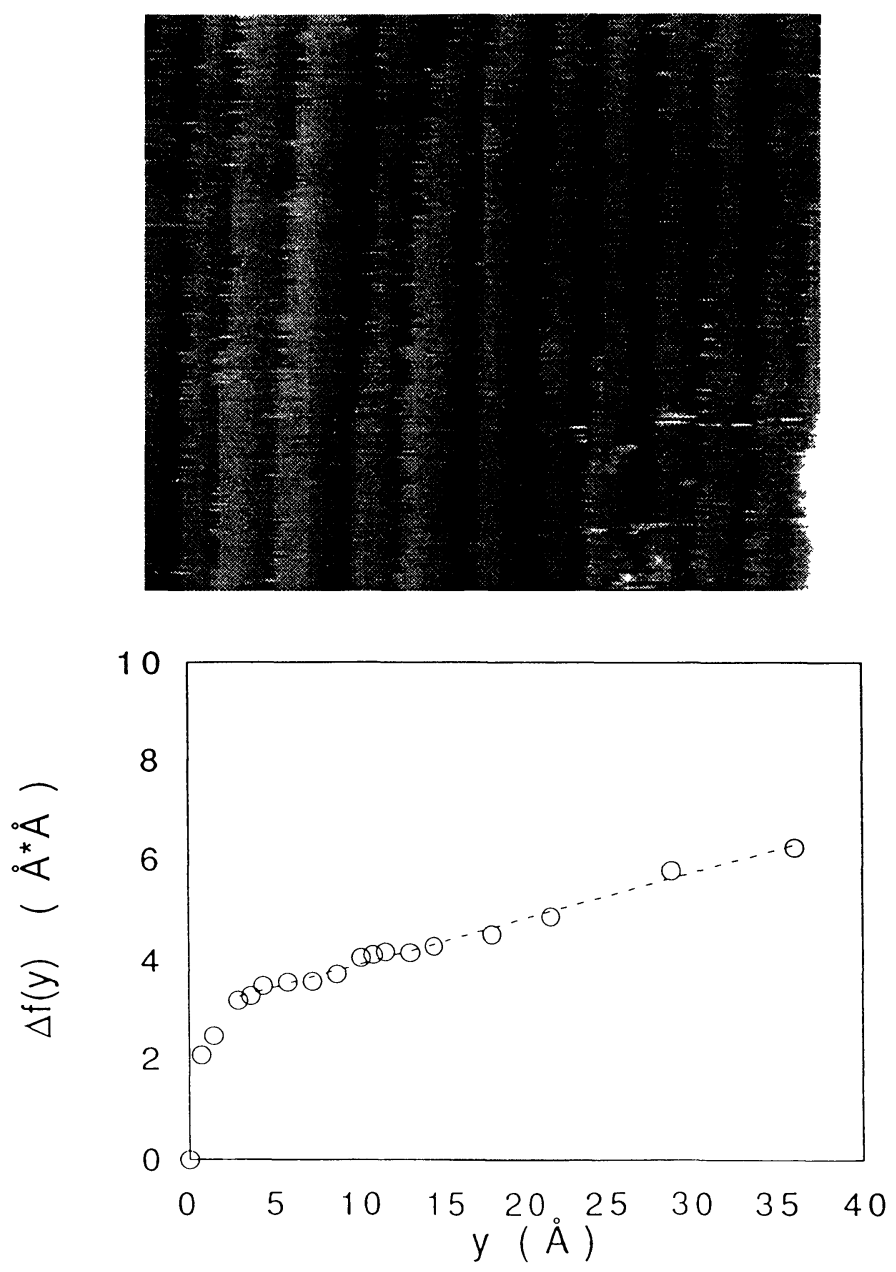

Fig. 3. $-184 \times 159 \AA$ image of the $\mathrm{Cu}(11,1,1)$ face with the corresponding correlation function for the terrace width. The data were acquired from left to right, scanning horizontally with a scanning frequency of $1 \mathrm{~Hz}$.

This is higher than the value of about $800 \mathrm{~K}$ which has been inferred from helium diffraction experiments [15], but similar to the results of recent STM studies $[16,17] . E_{\text {kink }}(810)$ is much smaller. This is in qualitative agreement with the more "wavy" appearance of the steps on image of figure 2 and can be related to the fact that creating kinks involves breaking of second neighbour bonds on [001] steps but stronger nearest neighbour bonds on [0-11] steps. The intensity A 
obtained for the interaction potential for the (810) face, that is for [001] steps, is $A=1,5 \times 10^{-30}$ J.m, comparable to the value $2.6 \times 10^{-30} \mathrm{~J} . \mathrm{m}$ obtained by helium diffraction [15] for [0-11] steps. The correlation length calculated from these values for the $(11,1,1)$ face is then comparable to the size of the image of figure 3, explaining the observed linearity of the correlation function for this image. Note that the order of magnitude for the interaction potential is the same for $\mathrm{Si}(111)$ where it was found $A=4.6 \times 10^{-30} \mathrm{~J} . \mathrm{m}$ [9].

This study shows that it is possible to extract quantitative information from STM images. One difficulty is the need to get good statistics. The values obtained here should be considered only as an order of magnitude, as they are obtained on too small an area. Possible directions for future studies include investigating different vicinal surfaces and their behaviour with temperature.

\section{Nucleation and annealing of ion bombardment induced defects on $\mathrm{Cu}(100)$.}

Steps also play a major role in the growth of crystals. Burton, Cabrera and Frank [18] showed that steps on a low index face of a crystal in equilibrium with its vapour are straight on the average, whereas on a surface in contact with a supersaturated (resp. undersaturated) vapor, steps should form closed loops, that is adatom (resp. vacancy) islands.

Low energy ion sputtering is, in some cases, closely analogous to evaporation in creating a highly supersaturated vacancy gas on the surface. $\mathrm{On} \mathrm{Cu}(100)$, these vacancies are very mobile at room temperature. They interact attractively and can eventually nucleate islands. A commercial ion gun (Riber) was used in this study. After the usual cleaning procedure, the $\mathrm{Cu}(100)$ surface was bombarded with $600 \mathrm{eV} \mathrm{Ar}^{+}$ions at room temperature. The flux and the fluence were monitored by measuring the neutralisation current on a small movable copper mass which could be positioned in front of the sample.

Vacancy islands can be observed on the surface as one monolayer deep areas bounded by monoatomic steps as shown in the image of figure 4. In addition, one also observes, in lesser number, adatom islands, all of monoatomic height, that appear brighter. This is predicted by numerical simulations [19], which show that an incident $\mathrm{Ar}^{+}$ion produces not only monovacancies, but also, in lesser number, adatoms on the surface. These adatoms are also very mobile at room temperature and can then nucleate adatom islands. Indeed, such islands have been observed by STM on Pt(111) after $600 \mathrm{eV} \mathrm{Ar}{ }^{+}$bombardment [20].

These islands are characterised by a unique shape -a square with rounded corners- and are all identically oriented. This shape is in qualitative agreement with theoretical predictions for the equilibrium shape of two dimensional islands on a square substrate [18]. According to these calculations, the sides of the islands are preferentially oriented along the close-packed rows of the (100) surface, this direction corresponding to the minimum of the free energy of the steps while the corners are rounded due to finite temperature entropic effects. This observation indicates that the surface mobility is high enough on this surface at room temperature for most of the islands to equilibrate, in agreement with recent STM estimates of the mobility of atoms along [110] steps on copper at room temperature $[1,16]$.

Pre-existing steps influence strongly the nucleation process, as shown by the images of figure 5, which were obtained after bombardment at different flux but identical fluence. The vacancy islands area corresponds to $0.3 \mathrm{ML}$ in both cases. It can be observed that the steps affect the island distribution in their vicinity. Island depletion zones are observable along the steps. The explanation is that the steps act as sinks for vacancies and adatoms, preventing nucleation in their vicinity. What is remarkable is that the efficiency of the annihilation processes of the vacancies or adatoms to steps strongly depends upon which step side is involved. For vacancy islands (Fig. 5a and 5b), depleted zones are located exclusively in the adjacent upper terrace of the steps. For the adatom 


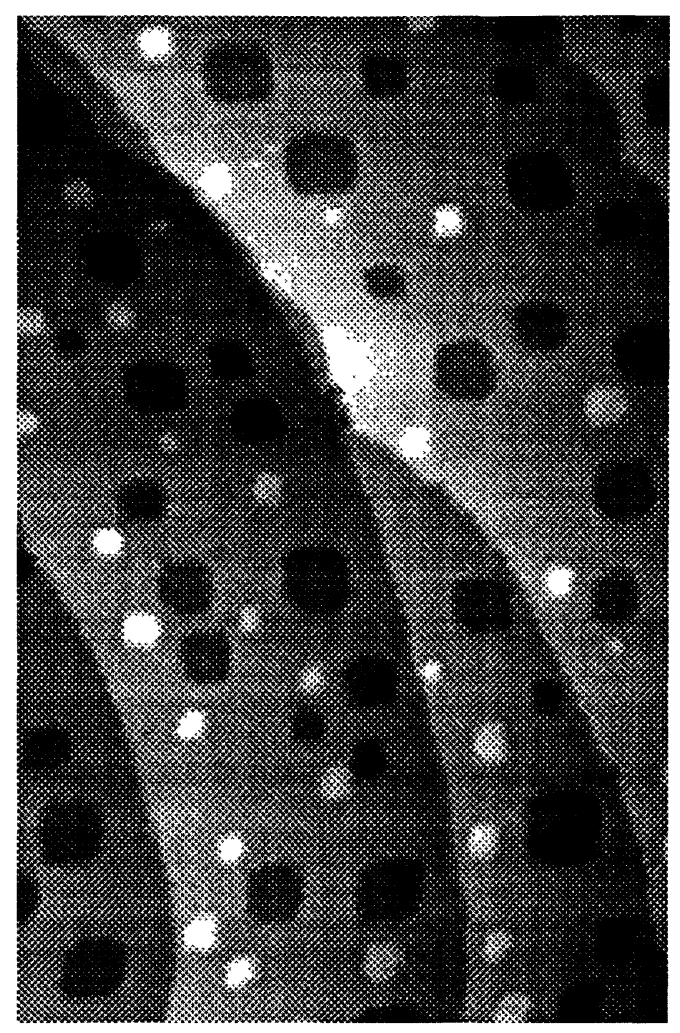

Fig. 4. $-960 \AA \times 1420 \AA$ image of the $\mathrm{Cu}(100)$ surface after bombardment at $300 \mathrm{~K}$ with $600 \mathrm{eV} \mathrm{Ar}^{+}$. The bombardment conditions were $1 \times 10^{14}$ ions $\mathrm{cm}^{-2} \mathrm{~s}^{-1}$ during $130 \mathrm{~s}$. Residual monoatomic steps run across the imaged area. Adatom and vacancy islands are visible on the (100) terraces.

islands (Fig. 5a), the symmetric feature holds: the islands depleted zones are exclusively seen in the adjacent lower terrace of the steps. Such an effect has been invoked by Schwoebel [22] as a possible origin for instabilities of step edges during step flow growth.

This can be connected with the mechanisms involved in mass transport during the abrasion process as suggested by Michely et al. [22]. At a "down step", a diffusing vacancy annihilates by exchange with a step edge atom. Both the vacancy and the step edge atom belong to the same layer so this mechanism requires only intralayer diffusion. At an "up step", the diffusing vacancy must be filled up by a step edge atom originating in the upper layer, implying interlayer diffusion. The asymmetry of the vacancy annihilation at steps demonstrates that an extra energetic barrier controls the interlayer diffusion. Similar arguments account for the adatoms behaviour. The adatom annihilation at an "up step" requires only intralayer diffusion whereas the annihilation at a "down step" requires interlayer diffusion.

Another striking feature appearing in figure 5 is that adatom islands are not visible in the image obtained with a high flux bombardment. This observation will be discussed in the following after presenting our results concerning the influence of the flux intensity on high fluence images. Figures $6 \mathrm{a}$ and $6 \mathrm{~b}$ show two images after exposure to similar fluence of 6 and $8 \times 10^{14}$ ions $\mathrm{cm}^{-2}$, but with flux intensity differing by nearly two orders of magnitude. The total area of the new uncovered terraces corresponds to the removal of about $0.6 \mathrm{ML}$ (Fig. 6a) and 0.8 ML (Fig. 6b). These 

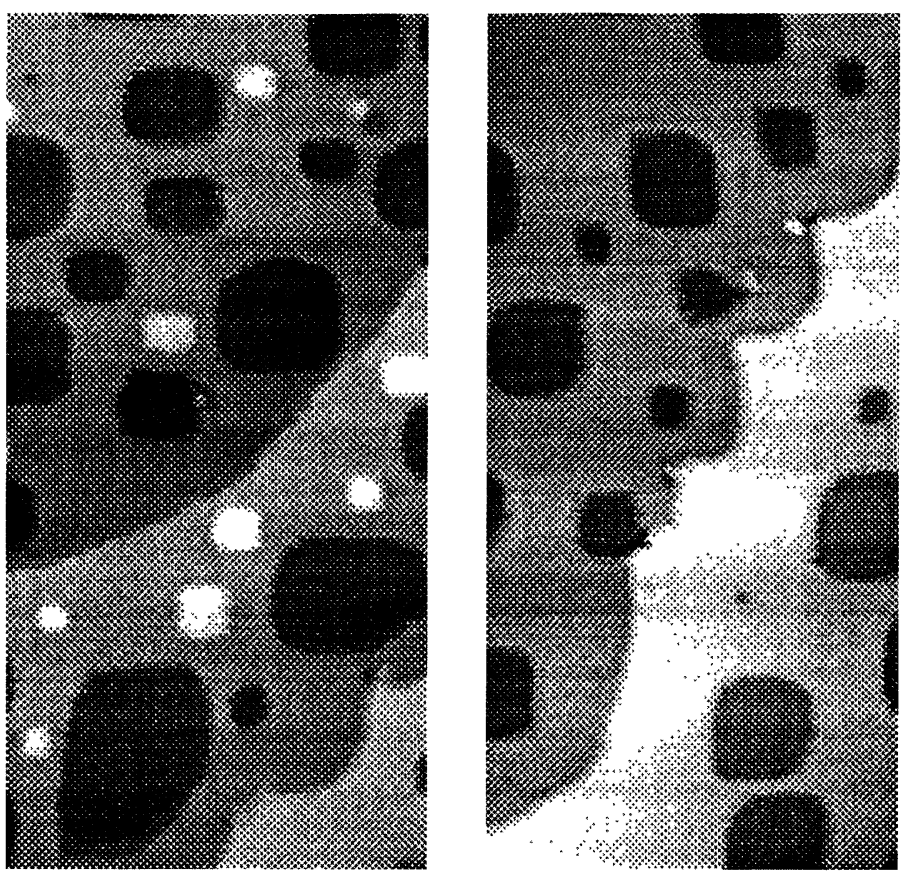

Fig. $5 .-630 \AA \times 1170 \AA$ images of the $\mathrm{Cu}(100)$ surface after bombardment at $300 \mathrm{~K}$ with $600 \mathrm{eV} \mathrm{Ar}^{+}$. The bombardment parameters were $1 \times 10^{12}$ ions $\mathrm{cm}^{-2} \mathrm{~s}^{-1}$ during $400 \mathrm{~s}$ for figure $2 \mathrm{a}$ and $1 \times 10^{14}$ ions $\mathrm{cm}^{-2} \mathrm{~s}^{-1}$ during $4 \mathrm{~s}$ for figure $2 \mathrm{~b}$. The total area of the vacancy islands corresponds to $0.3 \mathrm{ML}$.

data demonstrate clearly that the removal of matter moves from a three-dimensional (3D) to a quasi-ideal layer-by-layer regime as the flux increases. In the former case (Fig. 6a), four successive layers appear simultaneously uncovered. Several generations of vacancy islands are stacked within each other leading to the formation of $3 \mathrm{D}$ irregular negative pyramids. The observation of these structures implies that for bombardment with low ionic flux, the annihilation of vacancies to the surrounding "up step" border of the islands, i.e. the interlayer diffusion of matter, is inefficient. In the latter case (Fig. 6b), the initially exposed layer has been almost completely sputtered away before a second generation of vacancy islands starts to nucleate in the new uncovered layer. Thence under high flux bombardment all vacancies, whatever the layer in which they are created, contribute to the sputtering of the upper layer: a vacancy created in the lower layer is annihilated by an "up step" edge atom of the upper layer: interlayer diffusion does take place. The adatom islands which appear in figure $6 \mathrm{~b}$ are the remains of the outermost layer. So, the sputtering mode is clearly correlated to the flux of the impinging ions. An analogous behaviour has been previously inferred from TEAS measurement of the growth of Pt on $\mathrm{Pt}(111)$ [23]. A similar transition for sputtered $\mathrm{Pt}(111)$ by $600 \mathrm{eV}$ energy $\mathrm{Ar}^{+}$has been identified as a function of temperature [24].

Let us summarise these results: the influence of residual steps shows that the interlayer mass transport is controlled by an energetic barrier which limits its efficiency at step edges. The experiments with different flux demonstrate that, when it increases, the adatom islands disappear and the abrasion regime switches from $3 \mathrm{D}$ to $2 \mathrm{D}$.

Recent FIM experiments [25] and theoretical calculations [26] have been performed to investigate the diffusion of an adatom absorbed on the top of an island. To jump down across the surrounding step, the adatom has to overcome an extra energetic barrier in addition to the energy 

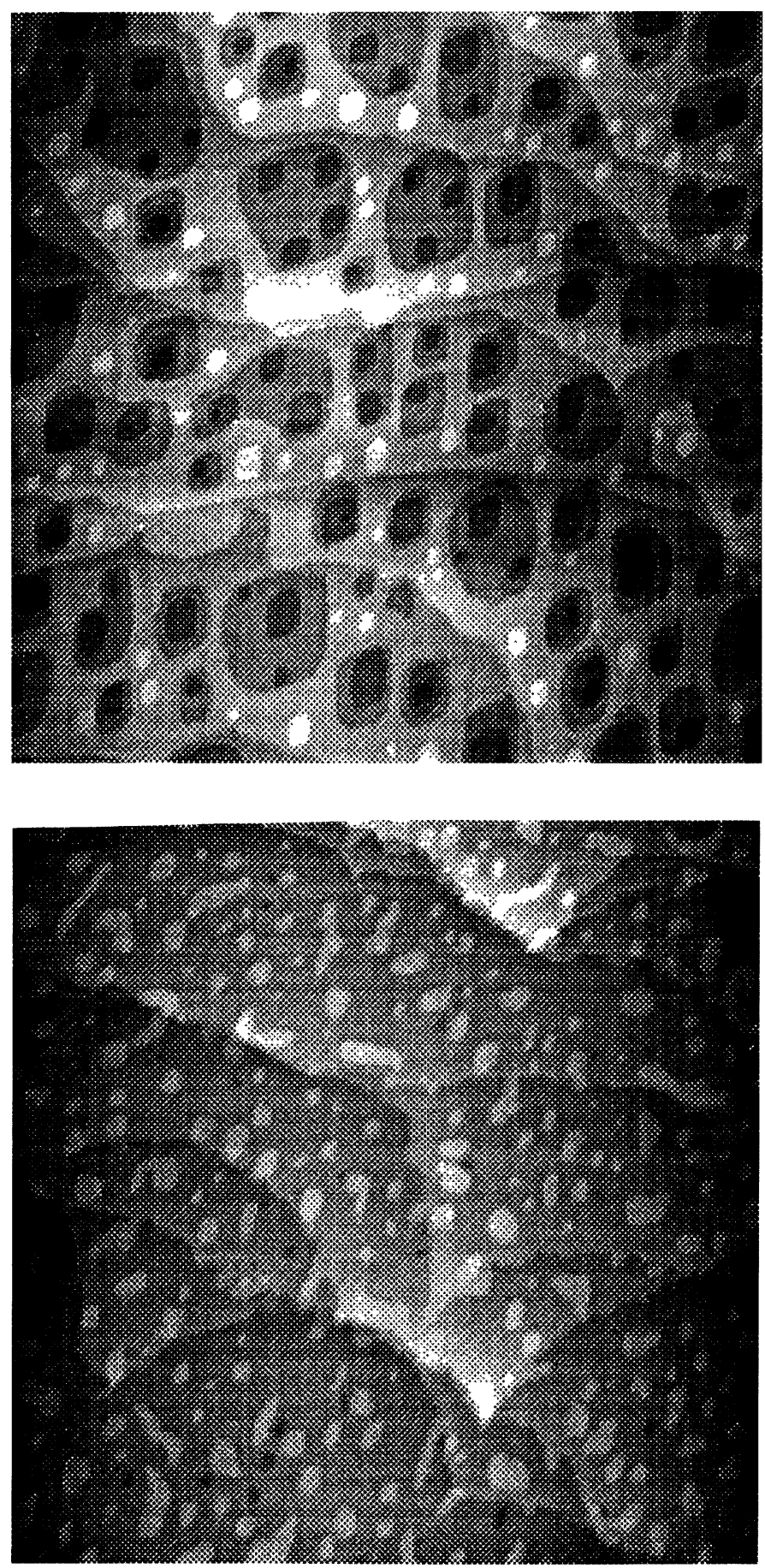

Fig. 6. $-2400 \AA \times 2600 \AA$ images of the $\mathrm{Cu}(100)$ surface after room temperature bombardment. Bombardment conditions: (a) $1.5 \times 10^{12}$ ions $\mathrm{cm}^{-2} \mathrm{~s}^{-1}$ during $400 \mathrm{~s}$, (b) $1 \times 10^{14}$ ions cm-2 $\mathrm{s}^{-1}$ during $8 \mathrm{~s}$. The coverage in vacancies is: in the surface layer 0.47 for (a) and 0.77 for (b), in the second layer 0.13 for (a) and 0.03 for (b), in the third layer 0.01 for (a) and 0.00 for (b). The adatom coverage is 0.05 for (a). 
of migration on a terrace. It has been suggested that such a barrier exists on many other metals. A quantitatively different but conceptually similar energetic barrier can be expected to control the interlayer transport of vacancies. We suggest, in the following, that our observations of the influence of the flux on the morphology of the surface can be understood in terms of this barrier.

The major consequence for the morphology of the surface of varying the flux is, according to standard theories of nucleation and growth [27], that a high number density of small islands is formed during high flux whereas a low number density of wide islands is formed under low flux sputtering. This is not what we observe: the mean island size does not seem to be strongly affected by the flux. Nevertheless, this is likely to be the consequence of the evolution of the surface during the time -at least 30 minutes- elapsed between the end of the bombardment and the imaging phase of the experiment [28]. Then, if one admits that a high number density of small islands is formed during high flux, our observations can be explained by assuming that the height of the interlayer diffusion barrier decreases for small enough islands. The absence of adatom islands at high flux stems then from the fact that the adatoms generated by the bombardment can recombine with vacancies at steps bounding the -small- vacancy islands. The $2 \mathrm{D}$ abrasion mode observed at high flux is then the direct consequence of the possibility for the vacancies generated inside a vacancy island to recombine with an atom of the surrounding terrace at the step bounding the island.

A similar size effect can be inferred from FIM results [25] and has also been invoked to explain the observations on the growth of $\mathrm{Pt}$ on $\mathrm{Pt}(111)$ mentioned above [23]. The critical size is then estimated to be around 10-20 $\AA$, well below the average island size we observed under high flux conditions.

These experiments are not only useful for a better understanding of ionic sputtering which is commonly used as a means to clean and process surfaces. The analogy mentioned above between this process and growth makes also this simple system of larger interest.

\section{Conclusions.}

These studies show that STM can give qualitative and in some cases quantitative insights in the mechanisms which are responsible for the morphology of surfaces. The knowledge of these mechanisms is often necessary as an input for the models which are designed to interpret the data obtained by more indirect techniques. In this respect, STM is complimentary to averaging techniques such as those based on diffraction.

\section{References}

[1] Girard J.C., Gauthier S., Rousset S., SACKS W., De CheVeigné S. and Klein J., to be published in Surf. Sci.

[2] Girard J.C., SAMSON Y., GaUthiER S., RouSSET S. and Klein J., to be published in Surf. Sci.

[3] KosSEL W., Nachrbl. Ges. Wiss. (Gottingen (1927) 135.

[4] RotTMAN C. and Wortis M., Phys. Rev. B29 (1984) 328.

[5] NozIERES P., in "Solid Far From Equilibrium", C. Godrèche Ed. (Cambridge Univ. Press, 1991).

[6] Gruber E.E. and Mullins W.W., J. Chem. Phys. Solids 28 (1967) 875.

[7] BARTELT N.C., EINSTEIN T.L. and WiLliams E.D., Surf. Sci. 240 (1990) L591.

[8] Balibar S., Guthmann C. and Rolley E., Surf. Sci. 283 (1993) 290.

[9] Alfonson C., Bermond J.M., Heyraud J.C. and MÉToIs J.J., Surf. Sci. 262, (1992) 371.

[10] Rolley E., Chevallier E., GuthmanN C. and Balibar S., preprint.

[11] FISHER M.E. and FISHER D.S., Phys. Rev. B25 (1982) 3192. 
[12] Wang X.-S., Goldberg J.L., BARTelt N.C., EinStein T.L. and Williams E.D., Phys. Rev. Lett. 65 (1990) 2430.

[13] Rousset S., Gauthier S., Siboulet O., Girard J.C., De Cheveigné S., Huerta-Garnica M., SACKS W., BELIN M. and KLEIN J., Ultramicroscopy $42-44$ (1992) 515.

[14] GIRARD J.C., Thèse, Université Paris 7 (1993).

[15] FABRE F., GoRSE D., LAPUjOULADE J. and SAlanON B., Europhys. Lett. 3 (1987) 737.

[16] Poengsen M., Wolf J.F., Frohn J., GieSEN M., IbaCh H., Surf. Sci. 274 (1992) 430.

[17] BARBIER L., COUSTY J., MASSON L. and SALANON B., private communication.

[18] Burton W.K., Cabrera N. and Frank F.C., Philos. Trans. Soc. London A 243 (1951) 299.

[19] WEBB R.P. and HARRISON D.E. Jr., Radiat. Eff. Lett. 86 (1983) 15.

[20] MiCHELY T. and COMSA G., Phys. Rev. B44 (1991) 8411.

[21] SCHWOEBEL R.L. and SHIPSEY E.J., J. Appl. Phys. 37 (1966) 3682.

[22] MiCHELY T., BESOCKE K.H. and COMSA G., Surf. Sci. 230 (1990) L135.

[23] Kunkel R., Poelsema B., Verheij L.K. and Comsa G., Phys. Rev. Lett. 65 (1990) 733.

[24] MICHELY T. and COMSA G., Surf. Sci. 256 (1991) 217.

[25] FINK H.W. and EHRLICH G., Surf. Sci. 143 (1984) 125.

[26] JARDIN J.P., DESJONQUĖRES M.C. and SPANJAARD D., J. Phys. C: Solid State Phys. 18 (1985) 1767.

[27] ZINSMEISTER G., Thin Solid Films 2 (1968) 497, Venables J.A., SPIller G.D.T. and Hanbücken M., Rep. Prog. Phys. 47 (1984) 399.

[28] We generally discern no evolution of the morphology of the sputtered surface on a time scale of about $30 \mathrm{mn}$, except in a few cases where the coalescence of two islands could be observed [2]. This does not exclude a fast evolution just after the end of the bombardment of the surface as the kinetics for this kind of system, being governed by surface diffusion, is expected to slow down rapidly with time. 